\title{
Clasificación de ecosistemas objeto de conservación en la zona costera del pacífico sur de Costa Rica
}

\section{Classification of ecosystems object of conservation in the coastal zone of the South Pacific of Costa Rica}

\author{
Juan Carlos Villegas-Arguedas ${ }^{1}$ \\ Doctorado en Ciencias Naturales para el Desarrollo, Costa Rica
}

\begin{abstract}
Resumen. Se clasificaron los polígonos de ecosistemas mediante fotointerpretación y análisis multiespectral en la zona costera de dos distritos del sur de Costa Rica, con la finalidad de identificar sitios sujetos de actividades para la conservación de la biodiversidad. Entre mayo y octubre 2016, se delimitaron y corroboraron en campo dichos ecosistemas. Los bosques se establecieron mediante fotointerpretación y establecimiento de puntos de verificación. En el caso de terrenos de aptitud forestal, se verificaron con medidas de inclinación porcentual promedio y el empleo de un inclinómetro de lectura óptica marca Suunto PM-5/1520; el parámetro mínimo en terrenos fue de más de $60 \%$ de pendiente, adicionalmente, la pedregosidad y la profundidad del suelo. Los humedales se obtuvieron/dispusierona partir de la existencia de vegetación hidrófita, suelos inundados o inundables y fauna asociada. La verificación de cada capa de ecosistemas se estableció gracias al índice de concordancia de Kappa-Cohen que utiliza las coberturas del suelo como modelo predictivo contra una red de puntos de ubicación geoespacial (GPS) de comprobación de campo, donde concordancias superiores al 70\% validan el modelo y la fotointerpretación. Se delimitaron 188 polígonos de cuatro tipos de ecosistemas, donde el bosque solo cubre 639.1 ha. El área total de estudio corresponde con 12223.10 ha., el mayor ecosistema natural es el manglar con 2808.64 ha. No se clasificaron terrenos de aptitud forestal sin bosque, mientras que los humedales correspondieron a 39 polígonos con un área total de 138.40 ha; y las asociaciones vegetales a humedales o manglares (AVHM), a 31 polígonos que cubren un área de 629.84 ha. Se concluye que estos ecosistemas naturales son alternativas de desarrollo sostenible para las comunidades con base en la actividad turística. Es recomendable que el Estado y las comunidades promuevan una agenda conjunta de desarrollo que permita aprovechar las ventajas de los ecosistemas naturales, también, se sugiere que los ecosistemas sujetos de conservación se incluyan como parte del Patrimonio Natural del Estado.
\end{abstract}

Palabras clave. Ecosistemas, zona costera, bosques, manglares, humedales, Costa Rica

\begin{abstract}
The ecosystem polygons were classified by photointerpretation and multispectral analysis in the coastal zone of two districts of the southern part of Costa Rica to identify sites subject to activities for the conservation of biodiversity. Between May and October 2016, these ecosystems were delimited and corroborated in the field. Forests were established by photointerpretation and establishment of verification points, in the case of land of forest aptitude, they were verified with measures of average percentage inclination by means of an optical reading inclinometer Suunto PM$5 / 1520$, land with more than $60 \%$ slope being the minimum parameter, in addition to stoniness and depth of the soil. Wetlands were determined from the existence of hydrophilic vegetation, flooded soils or flood areas and associated fauna. The verification of each ecosystem layer was determined from the Kappa-Cohen Concordance Index that uses the ground cover as a predictive model vs. a network of field verification geospatial location points (GPS), where concordances above $70 \%$ validate the model and the photointerpretation. 188 polygons from four ecosystem types were delimited, where the forest only covers 639.1 ha. The total area of study corresponds to $12,223.10$ ha. The largest natural ecosystem is the mangrove with 2,808.64 ha. Forest-free land was not determined without forest, while the wetlands correspond to 39 polygons with a total area of 138.40 ha. and vegetation associations to wetlands or mangroves (AVHM) correspond to 31 polygons covering an area of $629.84 \mathrm{ha}$. It is concluded that these natural ecosystems are alternatives for sustainable development for communities based on tourism. It is recommended that both the State and the communities promote a joint development agenda to take advantage of the benefits that these natural ecosystems provide. It is also recommended that ecosystems subject to conservation be considered as part of the State's Natural Heritage.
\end{abstract}

Keywords. Ecosystems, coastal zone, forests, mangroves, wetlands, Costa Rica 


\section{Introducción}

En Costa Rica, la zona marítimo-terrestre se integra/se compone de terrenos que pertenecen al Estado; esto significa que no son sujetos de apropiación por particulares. En estos terrenos, hay ecosistemas que, particularmente por legislación, las autoridades deben conservar (Ley 6043, 1977). En el caso de la propiedad privada fuera de esta zona marítimo-terrestre, presenta ecosistemas naturales, los cuales el Estado regula (Ley 7575, 1996).

En lo estatal y en lo privado, se deben identificar los diferentes ecosistemas, sean boscosos o humedales, para mejorar las acciones de conservación y de regulación en procura de mejorar la conservación de la biodiversidad, la cual es patrimonio de todos los ciudadanos.

Hoy día, la clasificación de las diferentes coberturas forestales o usos del suelo son comunes a partir de las herramientas de sensores remotos, sean imágenes por sobrevuelos, satélite, o georreferenciación en el campo, inclusive una combinación de datos digitales con comprobaciones en los sitios de interés, clasificación que conlleva una gran variedad de objetivos y razones (Silva, Bacao \& Caetano, 2017).

El uso del suelo tiene implicaciones legales y científicas, con aplicaciones en la seguridad alimentaria, la biodiversidad, la salud humana, el cambio climático o razones militares, lo cual conlleva la adecuada toma de decisiones a partir de dicho mapeo; son herramientas actuales del quehacer y conocimiento humano (Herold, See, Nandin-Erdene \& Fritz, 2016).

La caracterización de ecosistemas con sensores remotos es una técnica ampliamente utilizada en la investigación biológica y biogeográfica. Diferentes modelos se diseñan para obtenerla distribución de los ecosistemas o delos elementos de la naturaleza de interés, sean especies, biomas, cobertura forestal, humedales, bioclimas, entre muchos otros, o los cambios que, con el paso del tiempo, ocurren en estos (Popov, Kussul, Stankevich, Kozlova, Shelestov, Kravchenko, Korbakov \& Skakun, 2008; Ozdogan 2014).

Las técnicas de digitalización de información a partir de imágenes son variadas. Dichas imágenes se obtienen de diversas formas, a saber: satelitales, aéreas con instrumentos aerotransportados, drones o hasta con cámaras comunes desde lugares elevados. La digitalización será tan compleja como el instrumento utilizado y su precisión o tecnología, y los alcances de los objetivos de conservación dependerán de una adecuada identificación de los elementos naturales que dichas imágenes aporten, lo cual repercutirá en el diseño de la investigación y sus resultados (Fallas 2003).

En Costa Rica, existen diversas investigaciones sobre clasificación y distribución de ecosistemas o cambios en el uso del suelo y la cobertura forestal. Para la zona sur, Kappelle (2003) proporcionó una descripción y mapeo de los ecosistemas de la región, incluye ambientes naturales y antrópicos. De esta investigación, se derivan la descripción y la ubicación de 34 ecosistemas de la zona sur de Costa Rica. Esta descripción se derivó del trabajo a partir del uso de sensores remotos y detallados trabajos de campo con georreferenciación e inventarios de vegetación.

Una tarea/Una labor más detallada para un sitio en particular, en la zona sur de Costa Rica, fue desarrollado por el Centro de Alta Tecnología (CeNAT), específicamente, por el laboratorio del Programa de Investigaciones Aerotransportadas (PRIAS); es el Humedal Nacional TérrabaSierpe, área protegida adscrita bajo la protección y la tutela del Estado en el Sistema Nacional de Áreas de Conservación.

De este trabajo, el PRIAS (2010) estipuló/ acordó/fijó la existencia de 19 ecosistemas o categorías del uso del suelo como acuacultura, 
arroz, bancos de arena, bosques, costas rocosas, cultivos o plantaciones permanentes, islotes, lagos o lagunas, nubes, océano y ríos, palma aceitera, pantanos, pastos, plantaciones de banano, playas, suelos desnudos, vegetación asociada a los humedales "negra forra" (Acrostichum aureum) y yolillo (Raphia taedigera).

A pesar de los trabajos de Kappelle (2003) y PRIAS (2010), los factores de escala suelen ser elementos técnicos que, para una planificación más precisa o en lugares más específicos, pierden potencial en su uso; por lo tanto, para la zona marítimo-terrestre y el resto de la zona costera de los distritos de Bahía Ballena y Puerto Cortés, la presente investigación aporta precisión en identificación de estos ecosistemas naturales en una zona de menor escala como su principal objetivo. Esta pesquisa se desarrolló con la finalidad de identificar ecosistemas que, según sea su condición jurídica estatal-privada, podrían tener valor para generar actividades que conserven la biodiversidad; identificarlos y delimitarlos constituye un primer paso para su adecuado manejo.

\section{Metodología}

La investigación se desarrolló en la zona costera de los distritos Bahía Ballena y Puerto Cortés hasta el pie de monte, definido en la cota 20 m.s.n.m, para un área de estudio total de 12 223.10 ha , entre los $9^{\circ} 16^{\prime}$ Norte, $83^{\circ} 50^{\prime}$ Oeste y los $8^{\circ} 7^{\prime}$ Norte, $83^{\circ} 28^{\prime}$ Oeste.

Durante los meses de mayo y junio 2016, se delimitaron y clasificaron los diversos polígonos de cobertura forestal $u$ otros ecosistemas, como humedales y terrenos de aptitud forestal, mediante fotointerpretación de las imágenes de la Misión Carta 2010 de CeNATNASA y su visor en línea CENIGA (2016), además del sistema en línea de alta definición Google Earth con imágenes disponibles en la zona de estudio tipo QuickBird (2015) de
Digitalglobe, considerando las características de escala y de precisión de las imágenes (Díaz 2005, Claudet, García \& Lenfant, 2010).

El tipo deecosistema (bosque, humedal, terreno de aptitud forestal, manglares o asociaciones vegetales) se clasificó a partir de un análisis multiespectral que consideraba el espectro visible o la fisonomía de los diversos ecosistemas (Fallas 2003, Silva et al. 2017). Los polígonos constan de líneas imaginarias dibujadas sobre las imágenes, según ecosistema identificado, los cuales requirieron de ajustes y de recortes de acuerdo con la existencia de caminos públicos municipales o con la carretera Costanera Sur, por ende, se consideraron las reglas topológicas de rigor entre ellos. Los polígonos se trazaron estrictamente dentro del área definida como zona costera, entre la línea de pleamar y el pie de monte establecido.

Por un lado, para definir el área y la amplitud de la zona costera por evaluar, se utilizó la cartografía oficial del Instituto Geográfico Nacional.Por otra parte, se empleóla distribución político-administrativa de Costa Rica para definir los límites distritales. Así se estableció el área de estudio: desde la línea de costa hasta el pie de monte en la cota 20 m.s.n.m., con la ayuda de un Sistema de Información Geográfica (SIG) tipo ArcMap $10.1^{\mathrm{TM}}$ de ESRI@ (Enviroment Systems Reserch Institute Inc.) con proyección Lambert Sur para Costa Rica y Datum Nad27 Central.

Los terrenos de aptitud forestal que no tienen bosque se corroboraron en visitas de campo durante los meses de julio, setiembre y octubre 2016, a partir de puntos de verificación con medidas de inclinación porcentual promedio mediante un inclinómetro de lectura óptica marca Suunto PM-5/1520. El parámetro mínimo fue terrenos de más de $60 \%$ de pendiente, adicionalmente, la pedregosidad y la profundidad del suelo (de existir). 


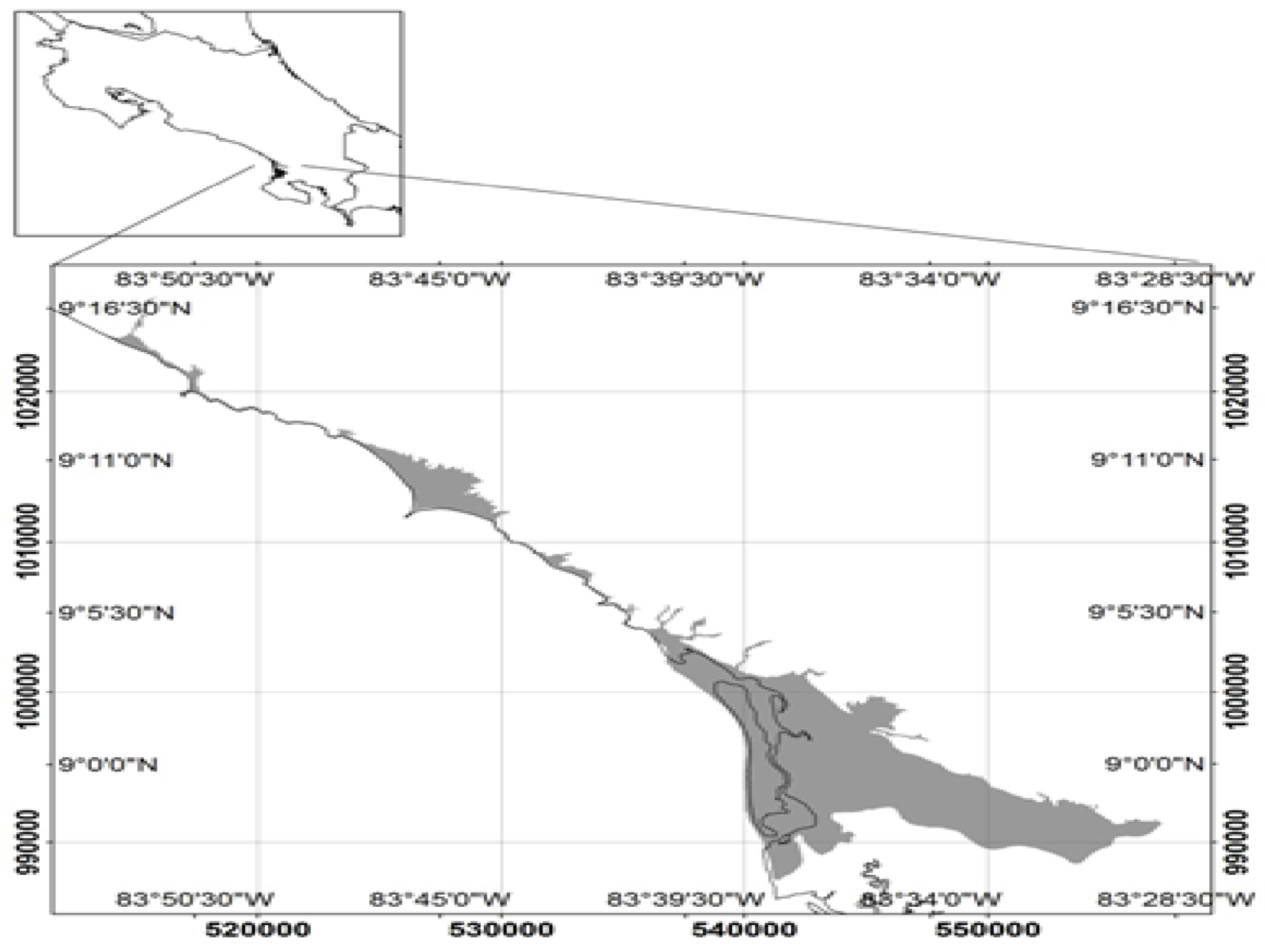

Figura 1. Ubicación del área de estudio

En el caso de los humedales que no son manglares, se definieron directamente en el campo en visitas durante los meses de julio, setiembre y octubre 2016, pues es difícil su discernimiento mediante imágenes. Para ello fue necesario, en primera instancia, la corroboración de su existencia en el inventario nacional de humedales; posteriormente en el campo, se delimitó a partir de la existencia de vegetación hidrófila, de los suelos inundados o inundables y de la fauna asociada. Estos humedales, una vez identificados en el campo, se delimitaron mediante un GPS, marca Garmin, modelo Map60 configurado para su uso en Lambert Sur para Costa Rica y Datum Nad27 Central, según Fallas (2008).

Para determinar los bosques, los humedales identificados, según la descripción anterior, y también,losmanglaresylosecosistemasasociados a los manglares, según la fotointerpretación y con la finalidad de garantizar que el tipo de hábitat y la cobertura forestal fotointerpretada sean 
correctos, se utilizó el índice de concordancia de Kappa-Cohen (Jenness \& Wynne 2007). Dicho índice evidencia la veracidad de un modelo predictivo (calidad de hábitat, cobertura forestal, distribución ecológica espacial, etc.) estimando su concordancia respecto a datos de corroboración de campo.

$$
\text { Kappa } k^{\prime} \frac{n \sum_{i=1}^{k} n_{i j}-\sum_{i=1}^{k} n_{i .} n_{. j}}{n^{2}-\sum_{i=1}^{k} n_{i .} n_{\cdot j}}
$$

Donde:

$\mathrm{n}=$ coberturas totales

$\mathrm{ni}=$ coberturas predictivas o supuestas

$n j=$ datos de comprobación de campo

En cuanto al establecimiento de los ecosistemas ubicados dentro del límite del área protegida Humedal Nacional Térraba-Sierpe, se utilizó como referencia los trabajos previos realizados por el Programa de Investigaciones Aerotransportadas (PRIAS) y su Laboratorio de Tecnologías de Información Geoespacial, en el marco del Proyecto Mapeo de Usos de la Tierra del Humedal Nacional Térraba-Sierpe (HNTS), con base en fotografías aéreas CARTA 2003 y 2005 (PRIAS 2010).

El criterio de validación del modelo predictivo se propone como mínimo en un valor de concordancia de $0.70(70 \%)$ de Kappa-Cohen, según el resultado de sus respectivas matrices de correlación (Jenness \& Wynne 2007) generadas por el software de Sistemas de Información Geográfica ArcView 3.3 ${ }^{\mathrm{TM}}$ de ESRIC (Enviroment Systems Reserch Institute Inc.) (Villegas 2012, 2013).

Como modelo predictivo, se utilizó la rasterización del uso del suelo fotointerpretado como se ha mencionado (grid digitalizado); se recalificó según la cobertura por verificar, sea bosque, terreno de aptitud forestal, manglar, humedal o vegetación asociada. Los nuevos raster (grids) consistieron de dos valores de acuerdo con la presencia (1) o ausencia (0) de la cobertura evaluada en el modelo predictivo que define el tamaño máximo de celdas en $100 \mathrm{~m} 2$ (Villegas 2012, 2013).

Los datos de comprobación de campo (puntos geoespaciales de verificación) se obtuvieron gracias a recorridos en visitas de campo durante los meses de julio, setiembre y octubre 2016, en los alrededores de los polígonos fotointerpretados y parte de su interior donde fue posible el recorrido; de igual forma que con el modelo predictivo (hábitats propuestos), a los puntos de verificación también se les asigna un nuevo campo que indicará la presencia (1) o ausencia (0) del tipo de cobertura o hábitat evaluado (Villegas 2012, 2013).

Varios son los factores que originan los impactos ambientales de la fragmentación de hábitats; se deben fijar la forma y el tamaño de parches, la distancia entre estos y su dispersión en el espacio. Entre los aspectos de forma con mayor impacto ecológico, está la exposición al "efecto de borde", el cual está determinado por la relación perímetro-área de los parches de ecosistema evaluado. Este último ecosistema se comporta de manera proporcional al aumento de la exposición del ambiente natural al lindero o borde de estos fragmentos y sus ecotonos, los cuales afectan negativamente a la vida silvestre a su interior (Bennet 1998).

Con la finalidad de demostrar el estado de la fragmentación de los ecosistemas entre distritos y poder evaluar más aspectos propios de los parches como el número de parches, su dispersión, densidad y superficie, se utilizó un índice de fragmentación, según Gurrutxaga (2003), el cual es una interfaz gráfica que facilita el análisis espacial de parches o fragmentos en el paisaje. A partir de los datos para cada bloque, se calculará el área del hábitat (CA), borde total 
(ET) y el promedio de la distancia al vecino más cercano $(\mathrm{MNN})$, insumos para el índice calculado mediante el software Patch Analyst de ArcView 3.3 ${ }^{\mathrm{TM}}$ de ESRIC) (Enviroment Systems Reserch Institute Inc.), según Elkie, Rempel \& Carr (1999), con base en

$F=$ superficie total del ecosistemalnúmero de parches del ecosistema $x$ dispersión de parches del ecosistema

Donde,

Dispersión de los parches del ecosistema (Rc) $=2 \mathrm{dc}(\lambda / \pi)$,

$\mathrm{dc}=$ distancia media en metros desde el borde de un parche hasta el borde del parche más cercano

$\lambda=$ densidad media de parches $=$ (número de parches/superficie total del área de estudio en ha) $\times 100=$ número de parches por cada 100 ha.

Según Gurrutxaga (2003), para cada bloque, el índice de fragmentación se regirá por su aumento o disminución, según sea su magnitud. El índice es inversamente proporcional en su valor; así, un aumento del índice se relaciona con una disminución del grado de fragmentación, y viceversa. Deacuerdoconla sintaxis dela fórmula, dicho aumento o disminución cuantitativa estará supeditado a la relación con la disminución de la superficie total de las manchas, un mayor o menor número de fragmentos (parches) o una mayor dispersión de estas.

Hipotéticamente, se esperó que la mayor cantidad y diversidad de bosques con menor fragmentación se encuentren en el distrito de Bahía Ballena, dada su mayor cercanía del pie de monte a la línea de costa y su menor presencia de aluviones utilizados para la agricultura extensiva. Por su parte, los manglares, los humedales y la vegetación asociada se encuentran, en mayor cobertura, en el distrito de Puerto Cortés, dada la presencia del Humedal Nacional Térraba-Sierpe.

\section{Resultados}

Delimitación y clasificación de los ecosistemas naturales en la zona costera de los distritos Bahía Ballena y Puerto Cortés.

Se estableció la cota 20 m s.n.m como límite máximo de elevación del pie de monte, la cual se utilizó para definir el área de estudio poligonal entre el océano, el pie de monte y los extremos este del río Barú y el oeste del río Grande de Térraba.

Se identificaron y delimitaron 188 polígonos de cuatro tipos de ecosistemas; en su mayoría, bosques (Tabla 1). El área total de estudio corresponde con 12 223.10, ha, donde la mayor cobertura corresponde con área sin elementos naturales para la conservación (8007.12 ha), mientras que el mayor ecosistema natural, representado en el área de estudio, es el manglar con 2808.64 ha (Tabla 1). No se determinaron terrenos de aptitud forestal sin bosque. Los humedales son 39 polígonos con un área total de 138.40 ha.

Se identificó un ecosistema de condiciones particulares el cual se denomina "asociaciones vegetales a humedales o manglares" (AVHM), ecosistema que tiene condiciones edáficas

Tabla 1

Cantidad y cobertura de los polígonos delimitados en el área de estudio

\begin{tabular}{llll}
\hline ECOSISTEMA & $\begin{array}{l}\text { N. }^{\circ} \\
\text { polígonos }\end{array}$ & Hectáreas & $\%$ \\
Bosque & 65 & 639.1 & 5.23 \\
Manglar & 52 & 2808.64 & 22.98 \\
Humedal & 39 & 138.4 & 1.13 \\
AVHM & 31 & 629.84 & 5.15 \\
ND & 1 & 8007.12 & 65.51 \\
& & 12223.1 & 100.00 \\
\hline
\end{tabular}

Fuente: elaboración propia 
propias, además de una composición vegetal sin especies de manglar ni especies adaptadas a inundaciones constantes o permanentes, de este tipo de ecosistema son 31 polígonos que cubren un área de 629.84 ha.

Caracterización de los ecosistemas naturales identificados y validación de su fotointerpretación.

Bosques. Los bosques corresponden con la mayor cantidad de polígonos identificados $(\mathrm{n}=$ 65) con un área de cobertura de 639.10 ha, de los cuales, 50 polígonos de bosque se ubican en el distrito Bahía Ballena (Figura 2); mientras que 15, en Puerto Cortés (Figura 3).

El índice de Kappa-Cohen sugiere una concordancia de $73 \%$ entre la fotointerpretación de los polígonos de bosques y los puntos de verificación ubicados en el campo ( $\mathrm{n}=323)$, lo que, según el criterio de validación del modelo predictivo, es correspondiente con la realidad en el terreno (Tabla 2, Figura 2).

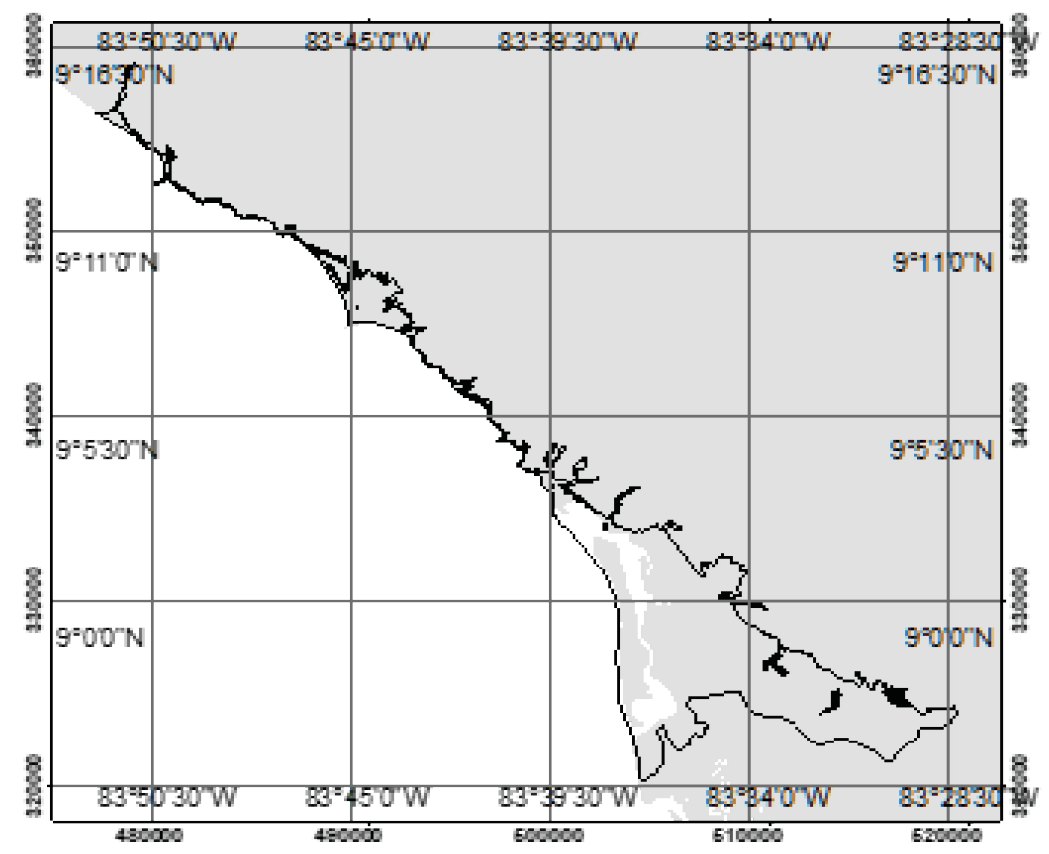

Figura 2. Ubicación de bosques en el área de estudio. Fuente: elaboración propia

Tabla 2

Matriz de correlación Kappa-Cohen para la cobertura bosque

\begin{tabular}{ccccc}
\hline Presencia-ausencia & N0 & N1 & \# puntos & \% concordancia \\
0 & 0.00 & 86.00 & 86.00 & 0.00 \\
1 & 0.00 & 237.00 & 237.00 & 1.00 \\
Puntos de & 0.00 & 323.00 & 323.00 & \\
verificación & & & & 73.00 \\
$\%$ concordancia & & 0.73 & & \\
\hline
\end{tabular}

Fuente. Datos propios 
Tabla 3

Cálculo del indice de fragmentación para los bosques de la zona de estudio

\begin{tabular}{cccccccccc}
\hline REGIÓN & MNN & $\begin{array}{c}\text { MN- } \\
\text { N/100=dc }\end{array}$ & NUMP & CA & CAT & $2 *$ MNN & Lambda & Rc & $\begin{array}{c}\text { ÍNDICE DE } \\
\text { FRAGMENTACIÓN }\end{array}$ \\
Ballena & 287.66 & 2.88 & 50 & 372.22 & 12223.10 & 575.32 & 0.41 & 0.75 & 9.93 \\
Cortés & 525.28 & 5.25 & 15 & 266.89 & 12223.10 & 1050.56 & 0.12 & 0.41 & 43.34 \\
& & & 65.00 & 639.11 & & & & & \\
\hline
\end{tabular}

$\mathrm{MNN}=$ distancia media entre parches

$\mathrm{dc}=$ distancia media en metros desde el borde de un parche hasta el borde del parche más cercano

NUMP = cantidad de parches

$\mathrm{CA}=$ superficie por zona de estudio individual o ecosistema

$\mathrm{CAT}=$ superficie total de toda la zona de estudio

Lambda: $\lambda=$ densidad media de parches = (número de parches/superficie total del área de estudio en ha) $\mathrm{x} 100=$ número de parches por cada 100 ha.

$\operatorname{Rc}=2$ dc $(\lambda / \pi)=$ Dispersión de los parches del ecosistema

Fuente: Datos propios.

Bahía Ballena presentó una mayor fragmentación de sus bosques (IF = 9.93), mientras que el distrito Puerto Cortés sugiere menor fragmentación, dado su mayor índice de fragmentación ( $\mathrm{IF}=43.34)$ (Tabla 3).

Manglares. Los manglares corresponden con la mayoría de hábitat natural en el área de estudio con 2808.64 ha. De los 52 polígonos de este ecosistema, 49 se ubican en el distrito de Puerto Cortés; mientras que solamente 3, en Bahía Ballena, de los cuales la mayor cobertura se localiza dentro del Parque Nacional Marino Ballena (Figura 3).

De acuerdo con el inventario nacional de humedales (MINAE, SINAC y UICN 1998), la mayoría de estos manglares están registrados. En el caso del distrito Bahía Ballena, el manglar en el Parque Nacional Marino Ballena es el denominado Estero Negro (Código 902), mientras que los polígonos en Puerto Cortés corresponden con los humedales de Térraba (código 905).

El índice de Kappa-Cohen sugiere una concordancia de $75 \%$ entre la fotointerpretación de los polígonos de mangle y los puntos de verificación ubicados en el campo $(n=20)$, lo que, según el criterio de validación del modelo predictivo, es correspondiente con la realidad en el terreno (Tabla 4, Figura 3).

Bahía Ballena presentó una mayor fragmentación de sus manglares (IF = 6.43), mientras que el distrito Puerto Cortés sugiere menor fragmentación dado su mayor índice de fragmentación (IF = 51.57) (Tabla 5).

Humedales. los humedales representan el ecosistema natural con menor área en la zona de estudio con 138.40 ha. De los 39 polígonos de este ecosistema, 10 se ubican en el distrito de Bahía Ballena, de los cuales la mayor cobertura se denomina Estero Changua-Uvita en el límite del Parque Nacional Marino Ballena, mientras que 29 están en Puerto Cortés (Figura 4).

De acuerdo con el inventario nacional de humedales (MINAE et al. 1998), los humedales registrados son Estero Changua-Uvita (código 901) y Ballena (código 903), los demás representarían registros nuevos. 


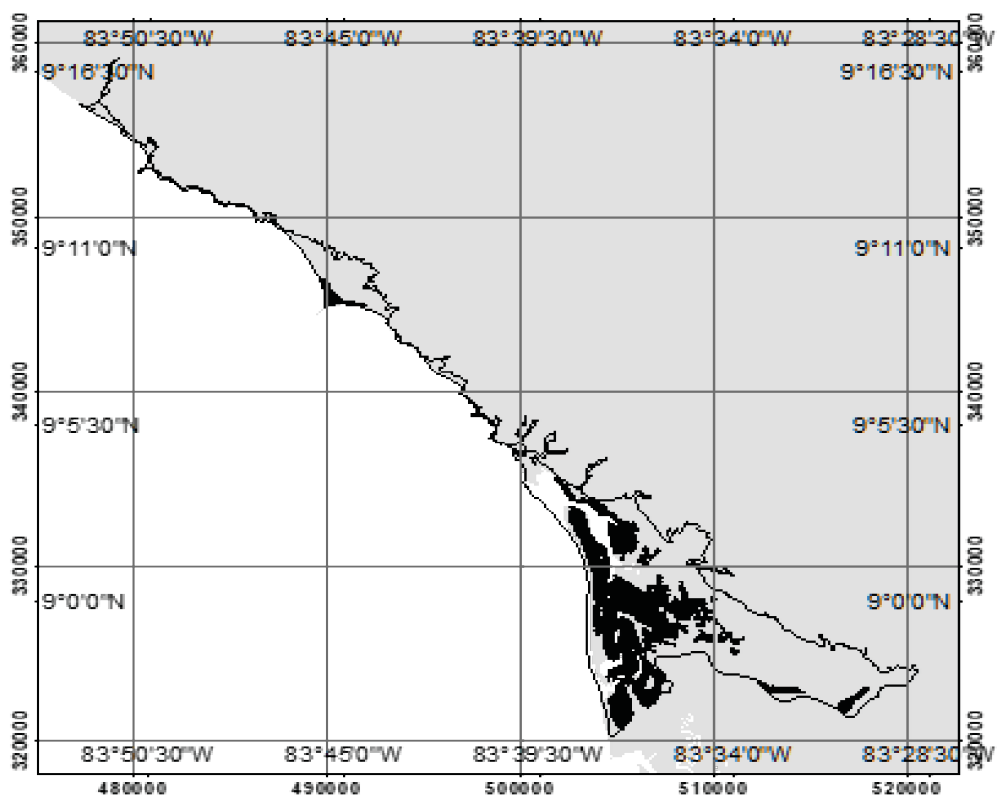

Figura 3. Ubicación de manglares en el área de estudio. Elaboración propia, 2017

Tabla 4

Matriz de correlación Kappa-Cohen para la cobertura manglares

$\begin{array}{ccccc}\text { Presencia-ausencia } & \text { N0 } & \text { N1 } & \text { \# puntos } & \text { \% concordancia } \\ 0 & 0.00 & 5.00 & 5.00 & 0.00 \\ 1 & 0.00 & 15.00 & 15.00 & 1.00 \\ \text { Puntos de verificación } & 0.00 & 20.00 & 20.00 & 75.00 \\ \text { \% concordancia } & & 0.75 & & \end{array}$

Fuente. Datos propios

Tabla 5

Cálculo del índice de fragmentación para los bosques de la zona de estudio

\begin{tabular}{cccccccccc}
\hline REGIÓN & MNN & $\begin{array}{c}\text { MN- } \\
\text { N/100=dc }\end{array}$ & NUMP & CA & CAT & $2^{*}$ MNN & Lambda & Rc & $\begin{array}{c}\text { ÍNDICE DE } \\
\text { FRAGMENTACIÓN }\end{array}$ \\
\hline Ballena & 11 & & & & 12 & & & & \\
& 332.86 & 113.33 & 5 & 94.97 & 223.10 & 22665.72 & .04 & 2.95 & 6.43 \\
Cortés & & & & & 12 & & & & \\
& 378.79 & 3.79 & 48 & 2345.12 & 223.10 & 757.58 & .39 & 0.95 & 51.57 \\
& & & 53.00 & 2440.09 & & & & &
\end{tabular}

$\mathrm{MNN}=$ distancia media entre parches. $\mathrm{dc}=$ distancia media en metros desde el borde de un parche hasta el borde del parche más cercano. NUMP = cantidad de parches. $\mathrm{CA}=$ superficie por zona de estudio individual o ecosistema. CAT = superficie total de toda la zona de estudio. Lambda: $\lambda=$ densidad media de parches $=$ (número de parches/superficie total del área de estudio en ha) x $100=$ número de parches por cada 100 ha. Rc $=2$ dc $(\lambda / \pi)=$ Dispersión de los parches del ecosistema. Fuente: Datos propios. 


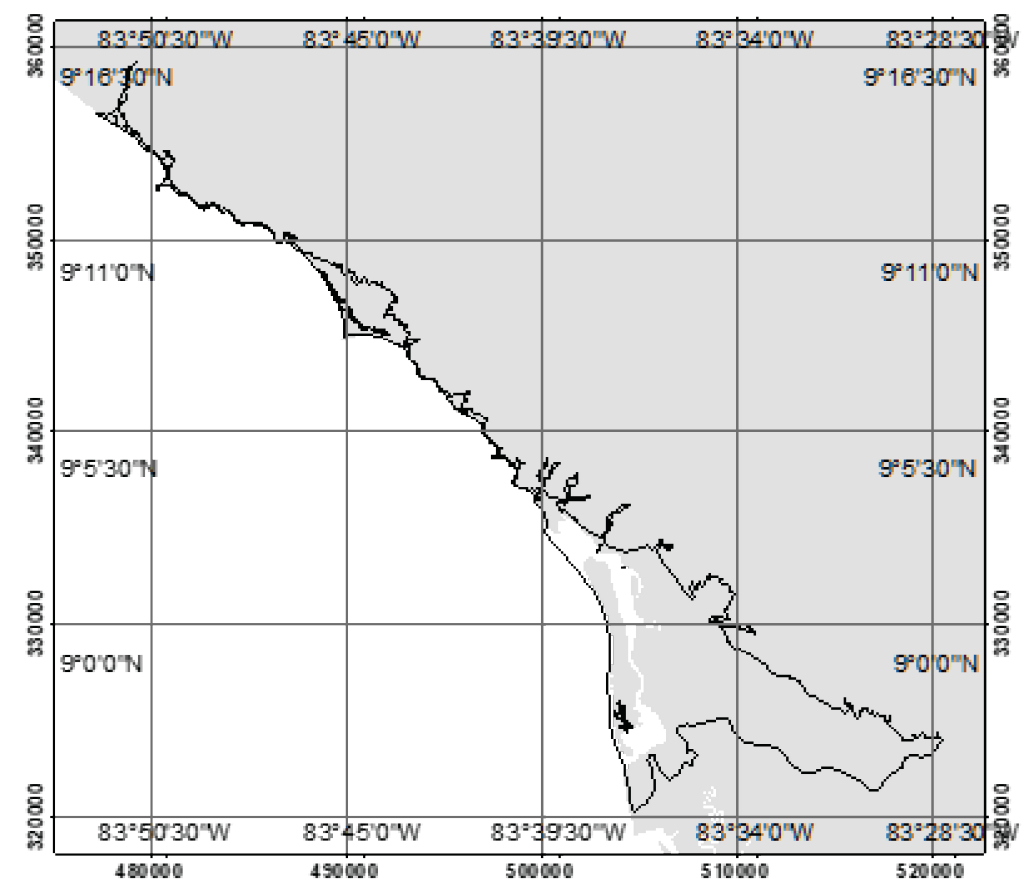

Figura 4. Ubicación de humedales en la zona de estudio. Elaboración propia, 2017

El índice de Kappa-Cohen sugiere una concordancia del $75 \%$ entre la fotointerpretación de los polígonos de humedales y los puntos de verificación ubicados en el campo $(\mathrm{n}=121)$, lo que, según el criterio de validación del modelo predictivo, corresponde con la realidad en el terreno (Tabla 6).

Bahía Ballena presentó una mayor fragmentación de sus humedales (IF $=1.61)$, mientras que el distrito Puerto Cortés sugiere menor fragmentación dado su mayor índice de fragmentación ( $\mathrm{IF}=2.13$ ); no obstante, para este ecosistema, la diferencia entre distritos es menor en comparación con los bosques y manglares (Tabla 7).

Vegetación asociada a manglares. Los ecosistemas con vegetación asociada a los manglares corresponden con 629.84 ha. Los 31 polígonos de esta cobertura natural se ubican en el distrito de Puerto Cortés (Figura 5).

Tabla 6

Matriz de correlación Kappa-Cohen para la cobertura humedales

\begin{tabular}{ccccc}
\hline Presencia-ausencia & N0 & N1 & \# puntos & \% concordancia \\
0 & 0.00 & 30.00 & 30.00 & 0.00 \\
1 & 0.00 & 91.00 & 91.00 & 1.00 \\
Puntos de verificación & 0.00 & 121.00 & 121.00 & \\
\% concordancia & & 0.75 & & 75.00 \\
\hline
\end{tabular}

Fuente. Datos propios 
Tabla 7

Cálculo del índice de fragmentación para los humedales de la zona de estudio

\begin{tabular}{cccccccccc}
\hline REGIÓN & MNN & $\begin{array}{c}\text { MN- } \\
\text { N/100=dc }\end{array}$ & NUMP & CA & CAT & $2 *$ MNN & Lambda & Rc & $\begin{array}{c}\text { Índice de } \\
\text { fragmentación }\end{array}$ \\
Ballena & 1183.66 & 11.84 & 31 & 95.47 & 12223.10 & 2367.32 & 0.25 & 1.91 & 1.61 \\
Cortés & 5126.49 & 51.26 & 9 & 46.16 & 12223.10 & 10252.98 & 0.07 & 2.40 & 2.13 \\
& & & 40.00 & 141.63 & & & & & \\
\hline
\end{tabular}

$\mathrm{MNN}=$ distancia media entre parches. $\mathrm{dc}=$ distancia media en metros desde el borde de un parche hasta el borde del parche más cercano. $\mathrm{NUMP}=$ cantidad de parches. $\mathrm{CA}=$ superficie por zona de estudio individual o ecosistema. $\mathrm{CAT}=$ superficie total de toda la zona de estudio. Lambda: $\lambda=$ densidad media de parches = (número de parches/superficie total del área de estudio en ha) $\times 100=$ número de parches por cada 100 ha. $\mathrm{Rc}=2 \mathrm{dc}(\lambda / \pi)=$ Dispersión de los parches del ecosistema.

Fuente: Datos propios.

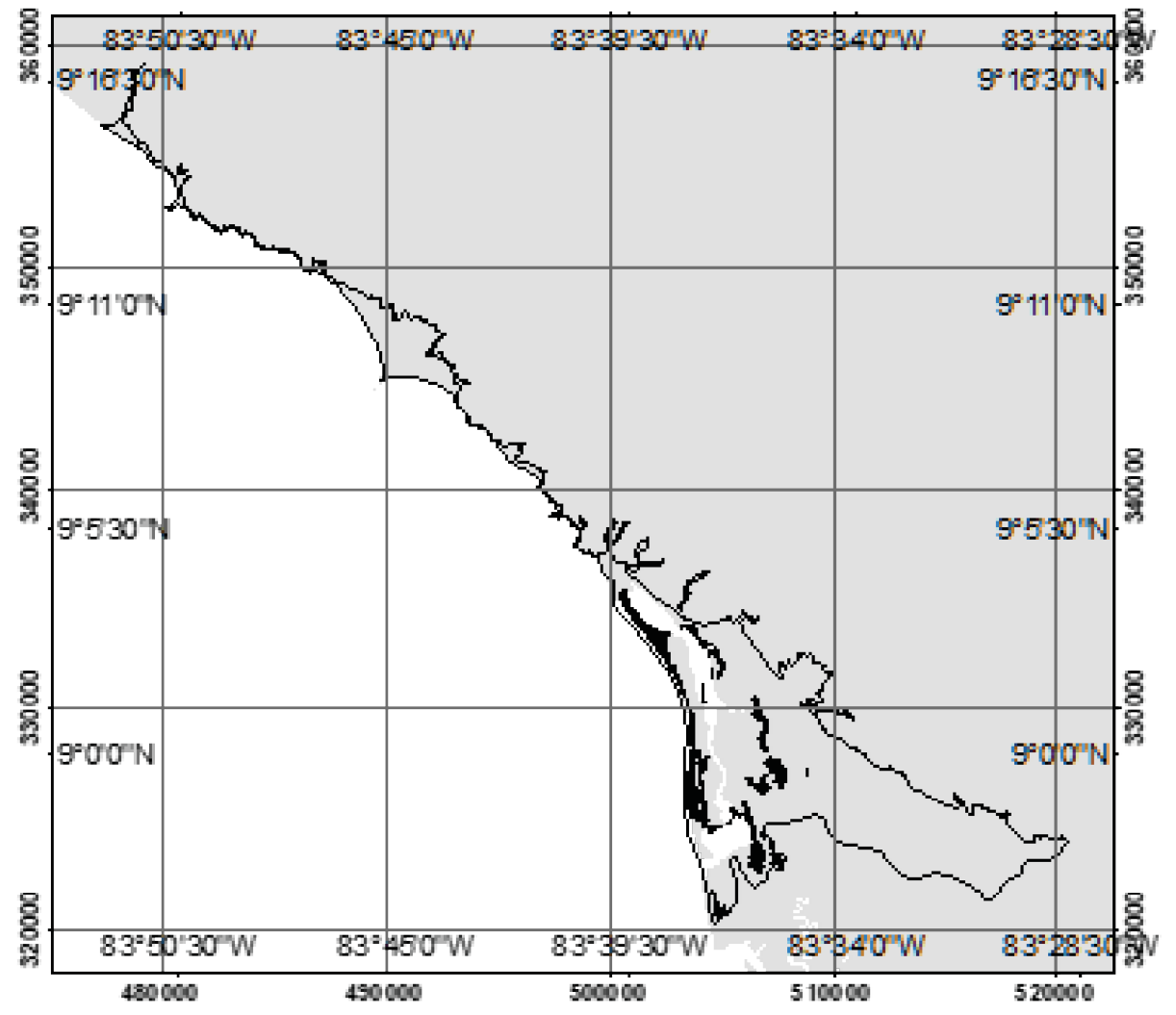

Figura 5. Ubicación de los polígonos de vegetación asociada a manglares en la zona de estudio. Elaboración propia, 2017 
El índice de Kappa-Cohen sugiere una concordancia de $86 \%$ entre la fotointerpretación de los polígonos de vegetación asociada a manglares y los puntos de verificación ubicados en el campo $(n=14)$, lo que, según el criterio de validación del modelo predictivo, es correspondiente con la realidad en el terreno
(Tabla 8). No hay interpretación para el índice de fragmentación entre distritos para este ecosistema, debido a que este solo se halla representado en el distrito de Puerto Cortés, asociado a los ecosistemas de manglar en el Humedal Nacional Térraba-Sierpe.

Tabla 8

Matriz de correlación Kappa-Cohen para la cobertura de vegetación asociada a manglares

\begin{tabular}{ccccc}
\hline Presencia-ausencia & N0 & N1 & \# puntos & \% concordancia \\
0 & 0.00 & 2.00 & 2.00 & 0.00 \\
1 & 0.00 & 12.00 & 12.00 & 1.00 \\
Puntos de verificación & 0.00 & 14.00 & 14.00 & 86.00 \\
\% concordancia & & 0.86 & & \\
\hline
\end{tabular}

Fuente. Datos propios

\section{Discusión}

Importancia de los ecosistemas identificados. El cantón de Osa, en la zona sur de Costa Rica, similar al resto del país, fue sometido al cambio de uso del suelo desde los tiempos de la colonia (PNUD 2012); por consiguiente, no es de extrañar que la mayor área en la zona de estudio corresponda con terrenos sin elementos naturales de conservación, son mayormente $(65,51 \%)$ áreas con terrenos de uso agropecuario.

En este orden de ideas, se destaca que alrededor de 32\% corresponde con áreas naturales como bosques, manglares o humedales con vegetación asociada a los manglares, lo que representa ecosistemas de alto valor para el desarrollo del cantón. Además, estas áreas constituyen elementos estratégicos para el incremento de actividades socioproductivas de valor ecológico (PNUD 2012) como el turismo, sector en el cual ya la región tiene experiencia demostrada en el manejo de las áreas protegidas circundantes, por ejemplo: la observación de ballenas u otros cetáceos, actividad fundamental en el desarrollo de estas comunidades costeras (Martínez-Fernández, Montero-Cordero y May-Collado 2011).

Los bosques de manglar son la segunda cobertura determinada $(22,98 \%)$, ecosistemas importantes también para la región, donde la mayoría están protegidos por el Sistema Nacional de Áreas de Conservación. Esto los hace menos vulnerables a los impactos de la tala o a su contaminación (Zamora y Cortés 2009; Benavides-Varela, Samper-Villareal y Cortés 2016), pero con posibilidad de uso controlado para las comunidades.

Además de los usos activos descritos, estos bosques protegidos de manglar representan elementos naturales de alto valor de conservación para la mitigación de los efectos del cambio climático, función conocida y documentada para estos ecosistemas (Yáñez-Arancibia, Day, Twilley y Day 2014). Dicha función constituye una ventaja para este cantón costero de Osa.

Respecto a los bosques costeros distintos a los manglares, se puede interpretar que, para la zona de estudio, significó una cobertura escasa con apenas 639.10 ha. Así se sugiere una fuerte 
disminución en esta zona costera, especialmente en el distrito de Puerto Cortés que mostró los mayores efectos e impactos negativos de la deforestación, evidenciado por escasa cobertura y representación forestal.

Bixby, Ulloa y Carrión (2000) reportaron que, en otros sectores de la zona sur de Costa Rica, en un lapso de 10 años, se deforestó hasta en 16\%. Los bosques se fragmentaron en 3\% y se cambió el uso del suelo a repastos para ganadería sobre todo. Llama la atención cómo estos cambios se dan a pesar de que el país cuenta con una legislación forestal fuerte y con presencia institucional consolidada del Gobierno en la región, con un acceso a la legalidad de la tenencia de la tierra con fuertes limitaciones. Por lo tanto, esto sugiere que la pérdida forestal ha ocurrido, principalmente, de manera ilegal (Franceschi 2006).

El mayor índice de fragmentación que presentó el distrito de Puerto Cortés se interpreta en la escasa representación de parches boscosos y su gran distancia entre parches; en consecuencia, su interpretación es inversa a la realidad estadística, análogamente con la interpretación de la fragmentación de los bosques en el distrito de Bahía Ballena. En este sector, se delimitaron mayor cantidad de parches, aunque de menores tamaños supeditados a la zona de estudio, lo que a su vez sugiere una mayor fragmentación dada su cercanía. Esto concuerda con la teoría de Gurrutxaga (2003) respecto a la interpretación de este índice, donde el incremento de la fragmentación se relaciona con la disminución de la superficie total de las manchas, un mayor número de fragmentos (parches) o una mayor dispersión de estas, interpretadas de manera inversamente proporcional al valor arrojado por la fórmula.

En este caso, aunque la mayoría de bloques se encuentran en el distrito de Bahía Ballena por la definición de la zona de estudio según el piedemonte y su cercanía a la costa, influyó que el índice fuera menor en este distrito, lo cual sugiere una mayor fragmentación. No pasa así en Puerto Cortés donde los bloques fueron pocos, pero de mayores tamaños y aislados, esto recuerda que cuanto menor el índice mayor es la fragmentación.

Considerando el ecosistema de humedales (sin incluir a los manglares y otras asociaciones vegetales asociados a ellos por analizarse separadamente), se desprende que, en primera instancia, los registros del Estado son limitados, dado que en esta investigación se sugieren 70 polígonos de coberturas del suelo con características de humedales, sean palustrinos, lacustrinos o ribereños.

De acuerdo con la verificación de la fotointerpretación, las coberturas del área de estudio tuvieron una concordancia de entre $75 \mathrm{y}$ $86 \%$, lo que valida su ubicación y determinación; sin embargo, es evidente que estos ecosistemas son delos más vulnerables y de fácil perturbación, ya que su mayoría se ubica en sitios con fácil acceso, rodeados de caminos, canales, cultivos y zonas pobladas.

Con excepción de los humedales lacustrinos y los asociados a ecosistemas de manglar en la Isla Garza en el área protegida Humedal Nacional Térraba-Sierpe, el resto tiene serias amenazas de desaparecer. Los humedales proveen de gran cantidad de servicios ecosistémicos como el mantenimiento del régimen hidrológico; son reservorios de agua y de biodiversidad acuática. También, gran cantidad de especies de aves, peces, anfibios y reptiles dependen, para su sobrevivencia, de estos ecosistemas, por ende, son parte de sus ciclos vitales y de reproducción, además de los servicios de captura de carbono, considerados los segundos ecosistemas más productivos en términos ecológicos del mundo (Bernal \& Mitsch 2013). 
Alternativas de manejo. Algunos ecosistemas naturales de los identificados y delimitados en esta investigación poseen ventajas para su conservación, particularmente, los que se encuentran en áreas protegidas estatales definidas en el cantón de Osa. Estos ecosistemas se ubicaron dentro del Parque Nacional Marino Ballena en el distrito Bahía Ballena y en el Humedal Nacional Térraba-Sierpe en el distrito Puerto Cortés.

Tales ecosistemas de bosque, manglares y humedales, en estas áreas protegidas, están sujetos para su uso a los respectivos planes de manejo y reglamentos de uso público. No así para los ecosistemas que se encuentran en terrenos privados o en la zona marítimo-terrestre sin planes reguladores municipales.

Certificación como patrimonio natural del Estado. El registro e inscripción de terrenos con elementos naturales de conservación a favor del Estado en zonas demaniales como la zona marítimo-terrestre se fundamenta en el uso de las facultades que se le confiere al Estado el artículo 140 inciso 3) de la Constitución Política.

Por su parte, existe una serie de instrumentos jurídicos concomitantes en relación con el tema de ecosistemas en las zonas costera, como la Ley General de Administración Pública, Ley 6227 del 2 de mayo de 1978; la Ley de Uso, Manejo y Conservación de Suelo 7779 del 30 de abril de 1998; el artículo 82 inciso a) de la Ley de Conservación de la Vida Silvestre 7317 del 30 de octubre de 1992; los artículos 22, 25 siguientes y 58 de la Ley de Biodiversidad, 7788 del 30 de abril de 1998, o los artículos 1, párrafo 2.ํ, 3.․ inciso i) 6 inciso a) y 13 párrafo 2. ${ }^{\circ}$, y 14 de la Ley Forestal 7575 del 13 de febrero de 1996.

Otros son el artículo 32, párrafo $2 .^{\circ}$ ) de la Ley Orgánica del Ambiente 7554 del 4 de octubre de 1995; la Ley Orgánica del Ministerio del Ambiente y Energía 7152 del 5 de junio de 1990; el artículo 3, incisos d y f, de la Ley del Servicio de Parques Nacionales 6084 del 24 de agosto de 1977, y los artículos 1, 3, 12 y 73 de la Ley sobre la Zona Marítimo Terrestre 6043 del 2 de marzo de 1977.

Existen más interpretaciones jurídicas sobre el Patrimonio Natural del Estado, como en los Dictámenes de la Procuraduría General de la República C-174-87 de fecha 8 de septiembre de 1987 y C-297-2004 de fecha 19 de octubre del 2004 donde se indica que el término "reservas equivalentes" engloba todas las áreas silvestres protegidas, supeditadas a planes de manejo que aseguren la adecuada protección, conservación y uso racional de los recursos naturales para un desarrollo sostenible, caso de los terrenos acá identificados en la zona costera.

Ese criterio se reitera en los dictámenes C-01588 de fecha 26 de enero de 1988; C-154-95 de fecha 7 de julio de 1995; C-191-96 de fecha 27 de noviembre 1996; C-026-2001 de fecha 7 de febrero del 2001; Opinión Jurídica OJ-062-2000 de fecha 9 de junio del 2000; y lo confirman los votos de la Sala Constitucional 5173-94, 1886-95, 1887-95 y 1998-01822, donde se establece que estas "reservas equivalentes" se regulan por su normativa y margina a las municipalidades de la administración y usufructo de estas (artículo 3. de la Ley de Zona Marítimo Terrestre). Es competencia de los órganos del Ministerio de Ambiente y Energía ejecutar los aspectos técnicos para el manejo y tutela de dichas áreas.

Por su parte, se destaca que la Ley sobre la Zona Marítimo Terrestre 6043 establece, en su artículo 1:

La zona marítimo-terrestre constituye parte del patrimonio nacional, pertenece al Estado y es inalienable e imprescriptible. Su protección, así como la de sus recursos naturales, es obligación del Estado, de sus instituciones y de todos los habitantes del país. Su uso y aprovechamiento están sujetos a las disposiciones de esta ley. 
Todoecosistema natural sujeto de conservación por ley delimitado como patrimonio natural del Estado en la zona marítimo-terrestre por jurisdicción es administrado por la figura estatal del Sistema Nacional de Áreas de Conservación, institución que las puede declarar formalmente áreas protegidas o ampliar con estas zonas otras ya establecidas. Esto se ratificó en el Dictamen C-297-2004 de la Procuraduría General de la República de Costa Rica, donde, a su vez, el Estado, por razones de conveniencia, oportunidad y costos, considera necesario la delimitación no solamente de los ecosistemas de bosques, sino de los otros ecosistemas que integran el patrimonio natural del Estado, ubicados dentro de la zona marítimo-terrestre.

Todo lo anterior repercute en posteriores estrategias e instrumentos de planificación y de manejo de las zonas costeras en Costa Rica, como son los planes reguladores costeros, definidos por las respectivas municipalidades. Para la zona de interés, corresponde a la Municipalidad de Osa, por lo que previo a la definición de las zonas de uso en estos terrenos demaniales, las municipalidades deberían contemplar, en primera instancia, los ecosistemas definidos en este estudio para propiciar su conservación.

Fomento del turismo como actividad socioproductiva: en Costa Rica, la visitación turística ha mantenido un incremento exponencial desde la década 1960, se han registrado más de dos millones y medio de turistas en la actualidad (Vargas-Ulate, 2009). De estos, más de $80 \%$ ha visitado en promedio cinco áreas protegidas estatales, cuyos intereses han sido, principalmente, la observación de biodiversidad (aves, mamíferos, reptiles), caminatas por los senderos y la toma del sol para disfrutar la belleza escénica de las playas costarricenses (Vargas-Ulate, 2009).

En la zona sur del país, la pérdida de la cobertura forestaly delasáreas verdes disminuye, a su vez, las posibilidades de espacios para la recreación y el turismo. Asimismo, el disfrute de la belleza escénica se ve seriamente diezmado con el cambio de uso del suelo hacia actividades con limitado valor agregado, verbigracia: la ganadería o los cultivos de gran extensión como arroz o palma aceitera.

Aunque no se dispone de registro de ingresos al Humedal Nacional Térraba-Sierpe, más de un millón ochocientos mil turistas visitaron áreas protegidas durante el 2016 en Costa Rica.

Los parques nacionales costeros del Pacífico Manuel Antonio y Marino Ballena son dos de las tres áreas protegidas más visitadas (Sinac, 2016). Por lo tanto, se hace imperativo que, en todos los demás espacios naturales determinados, sean bosques, manglares o humedales que no se encuentran en las áreas protegidas Marino Ballena o Térraba-Sierpe, la municipalidad de Osa y los pobladores establezcan un plan de manejo turístico sostenible que permita aprovechar los diversos recursos naturales con los que disponen y fomenten políticas de desarrollo comunal relacionadas con la actividad turística.

El Sistema Nacional de Áreas de Conservación tiene una gran responsabilidad en el manejo de estos ecosistemas, estén en zonas demaniales del Estado; en propiedad privada dada su competencia en el control y manejo forestal y resguardo a los humedales; o como se ha recalcado en la administración de las dos grandes áreas protegidas, que se encuentran una en el distrito Bahía Ballena y otra en el distrito Puerto Cortés.

En suma, la municipalidad tiene la administración de las zonas no consideradas patrimonio natural del Estado en los terrenos estatales, mientras que, en propiedades privadas, podría brindar acompañamiento para promocionar mejores prácticas agrícolas que 
promuevan la conservación o la reforestación, de ser posible.

\section{Conclusión}

Más que representar obstáculos para el desarrollo de los distritos de Bahía Ballena o Puerto Cortés, los ecosistemas naturales acá identificados y delimitados son alternativas de desarrollo sostenible para estas comunidades, donde, como se ha sugerido, el incremento de la actividad turística requiere un uso más equilibrado ambiental y socialmente, a fin de que se beneficien las comunidades, los turistas y sobre todo el ambiente.

Una cuarta parte de esta zona de estudio, en estos distritos costeros, aún tiene elementos naturales importantes para la conservación, por ende, debe procurarse su conservación y manejo adecuado. Particularmente, los bosques de manglar que representa la cobertura forestal más amplia en la zona estudiada. A su vez, debe ponerse atención a la gran cantidad de humedales que no se habían inventariado por parte de las autoridades ambientales, ecosistemas frágiles y de fácil degradación, los cuales deben protegerse y, en la medida de lo posible, declararse formalmente como patrimonio natural del Estado y asignarles categoría formal de conservación como áreas protegidas.

No solo la identificación y delimitación de los ecosistemas descritos en esta investigación se requiere para garantizar su conservación, estos elementos naturales necesitan de una caracterización que permita conocer su "salud"; cuál es la flora y fauna asociada a éstos; y una mejor identificación de los potenciales para el desarrollo de las comunidades aledañas. A partir de esta definición de tales ecosistemas, es factible realizar investigaciones ambientales y sociales que coadyuven a un mejor entendimiento del potencial de uso y de las ventajas de su conservación y manejo.
Las instituciones del Estado (el Sistema Nacional de Áreas de Conservación, las municipalidades, el Instituto de Desarrollo Rural, el Instituto Costarricense de Turismo o el Ministerio de Agricultura y Ganadería) deben promover una agenda conjunta, en la cual las comunidades de Bahía Ballena, Ojochal, Sierpe, Puerto Cortés, entre otras, así como sus pobladores puedan aprovechar las ventajas que estos ecosistemas naturales les proporcionan.

La visualización de estos ecosistemas naturales en la zona estudiada como objetivo de esta investigación permite ampliar el panorama de las oportunidades futuras para estas comunidades inmersas en esta matriz de usos de la tierra, y sobre todo, precisa los sitios que requieren atención por parte de las autoridades para evitar la pérdida irreparable de estos reservorios de la biodiversidad, en dos distritos costeros de la zona sur de Costa Rica.

\section{Agradecimientos}

A la Universidad Nacional de Costa Rica, por permitirme desarrollar este proyecto de estudios doctorales, en su programa DOCINADE; y al Área de Conservación Osa del SINAC, por brindar los permisos para esta investigación y el apoyo técnico de sus funcionarios.

\section{Referencias}

Asamblea Legislativa. Gobierno de Costa Rica (2 de marzo de 1977). Ley sobre la Zona Marítimo Terrestre 6043. La Gaceta.

Asamblea Legislativa. Gobierno de Costa Rica (24 de agosto de 1977). Ley del Servicio de Parques Nacionales 6084. La Gaceta.

Asamblea Legislativa. Gobierno de Costa Rica (2 de mayo de 1978). Ley General de Administración Pública Ley 622. La Gaceta.

Asamblea Legislativa. Gobierno de Costa Rica (5 de junio de 1990). Ley Orgánica del Ministerio del Ambiente y Energía 7152. La Gaceta. 
Asamblea Legislativa. Gobierno de Costa Rica (30 de octubre de 1992). Ley de Conservación de la Vida Silvestre 7317. La Gaceta.

Asamblea Legislativa. Gobierno de Costa Rica (4 de octubre de 1995). Ley Orgánica del Ambiente 7554.La Gaceta.

Asamblea Legislativa. Gobierno de Costa Rica (13 de febrero de 1996). Ley Forestal $7575 \mathrm{La}$ Gaceta. Gobierno de Costa Rica (30 de abril de 1998). Ley de Uso, Manejo y Conservación de Suelo 7779. La Gaceta.

Asamblea Legislativa. Gobierno de Costa Rica (30 de abril de 1998). Ley de Biodiversidad, 7788. La Gaceta. Bennet, A. F. (1998). Linkages in the landscape: The role of corridors and connectivity in wildlife conservation. Gland, Suiza: IUCN..

Benavides-Varela, C.; Samper-Villareal, J. y Cortés, J. (2016). Cambios en la cobertura de manglares en Bahía Culebra, Pacífico Norte de Costa Rica (1945-2010). Rev. Biol. Trop., 64(3): 955-964.

Bernal, B. \& Mitsch, W. J. (2013). Carbon sequestration in freshwater wetlands in Costa Rica and Botswana. Biogeochemistry (2013), 115: 77-93.

Bixby, L. R.; Ulloa, T. M., y Carrión, R. B. (2000). Capítulo 16. Bosque y Población en la Península de Osa. Población del Istmo 2000, 335.

Claudet, J.; García, J. A. \& Lenfant, P. (2010). Combined Effects of Levels of Protection and Environmental Variables at Different Spatial Resolutions on Fish Assemblages in a Marine Protected Area. Conservation Biology, 25(1): 105-114.

Centro Nacional de Información Geo-Ambiental (CENIGA) (2016). Visor geográfico. Recuperado de http://www.sinac.go.cr/ceniga/?q=content/visor-ceniga
Díaz, J. M. (2005). Esquemas espaciales de zonación ecológica y morfología de las lagunas de los atolones y complejos arrecifales de un archipiélago oceánico del Caribe: San Andrés y Providencia (Colombia). Rev. Acad. Colomb. Cienc. 29(112).

DigitalGlobe (2015). Imágenes tipo satellite QuickBird. Recuperado de http://www.digitalglobe.com

Elkie, P.; Rempel, R. \& Carr, A. (1999). Patch Analyst User's Manual. Ont. Min. Natur. Resour. Northwest Sci. \& Technol. Thunder Bay, Ontario, Canada. 16 p +Append.

Fallas, J. (2003). Teledetección espacial. Laboratorio de teledetección y sistemas de información geográfica. Mimeografiado. Heredia, Costa Rica: EDECA-PRMVS-UNA

Fallas, J. (2008). Proyecciones cartográficas y datum. Laboratorio de teledetección y sistemas de información geográfica. Mimeografiado. Heredia, Costa Rica: EDECA-PRMVS-UNA

Franceschi, H. (2006). Conflictos socioambientales intercampesinos por los recursos naturales. Rev. Ciencias Sociales, 111-112: 37-56 (I-II).

Gurrutxaga-San Vicente, M. (2003). Índice de fragmentación y conectividad para el indicador de biodiversidad y paisaje de la Comunidad Autónoma del País Vasco. Vasco, España: $\underline{\mathrm{IKT}}$. País.

Herold, M.; See, L.; Nandin-Erdene, T. \& Fritz, S. (2016). Towards an Integrated Global Land Cover Monitoring and Mapping System. Rev. Remote Sens.8, 1036.

Jenness, J. \& Wynne, J. (2007). Cohen's Kappa an classification table metrics 2.1: An ArcView $3 x$ extension for accuracy assessment of spatially-explicit models. USGS - Southwest Biological Science Center Colorado Plateau Research Station. 
Kappelle, M. (2003). Ecosistemas del Área de Conservación Osa (ACOSA). Volumen 2 of Serie Ecosistemas de Costa Rica. University of Texas. INBio, San José, Costa Rica.

Martínez-Fernández, D.; Montero-Cordero, A. y May-Collado, L. (2011). Cetáceos de las aguas costeras del Pacífico norte y sur de Costa Rica. Rev. Biol. Trop. 59(1): 283-290.

Ministerio del Ambiente y Energía (MINAE). Costa Rica, Sistema Nacional de Áreas de Conservación (SINAC). Unión Internacional para la Conservación de la Naturaleza, (UICN), Regional Office for Meso-America, Netherlands, Córdoba-Muñoz, R.; Romero-Araya, J. C. y Windevoxhel-Lora, N. J. Eds. (1998). Inventario Nacional de Humedales. IUCN Publication. San Jose: UICN ORMA, 380p. : maps. ISBN: 9968-743-21-6.

Ozdogan, M. (2014). A Practical and Automated Approach to Large Area Forest Disturbance Mapping with Remote Sensing. Rev. PLOS ONE 9(4): e78438. doi:10.1371/journal. pone.0078438.

Popov, M. A.; Kussul, N. N.; Stankevich, S. A.; Kozlova, A. A.; Shelestov, A. Y.; Kravchenko, O. M.; Korbakov, M. B. \& Skakun, S. V. (2008). Web service for biodiversity estimation using remote sensing data. International Journal of Digital Earth 1(4): 367-376.

Procuraduría General de la República de Costa Rica (8 de setiembre de 1987). Dictamen C-174-87.

Procuraduría General de la República de Costa Rica (26 de enero de 1988). Dictamen C-01588.

Procuraduría General de la República de Costa Rica (7 de julio de 1995). Dictamen C-154-95.

Procuraduría General de la República de Costa Rica (27 de noviembre de 1996). Dictamen C-191-96.
Procuraduría General de la República de Costa Rica (9 de junio de 2000). Opinión Jurídica OJ-062-2000.

Procuraduría General de la República de Costa Rica (7 de febrero de 2001). Dictamen C-0262001.

ProcuraduríaGeneraldelaRepúblicadeCostaRica (19 de octubre de 2004). Dictamen C-297-2004.

Programa de Investigaciones Aerotransportadas (PRIAS) (2010). Reporte técnico: Proyecto Mapeo de usos de la tierra del Humedal Nacional TérrabaSierpe (HNTS) basado en Fotografías aéreas CARTA 2003 y 2005. Laboratorio de Tecnologías de Información Geoespacial. Mimeógrafo.

Programa de las Naciones Unidas para el Desarrollo (PNUD) (2012). Plan de desarrollo humano local. Cantón de Osa 2013-2023. Mimeógrafo. Osa, Costa Rica.

Sala Constitucional de la República de Costa Rica (9 de setiembre de 1994). Voto 5173-94. Sentencia: 05173. Expediente: 94-001009-0007CO. Fecha: 09/09/1994. Hora: 09:42:00 a.m. Emitido por Sala Constitucional.

Sala Constitucional de la República de Costa Rica (7 de abril de 1995). Voto 1886-95. Sentencia: 01886. Expediente: 91-003145-0007-CO. Fecha: 07/04/1995. Hora: 09:12:00 a.m. Emitido por Sala Constitucional.

Sala Constitucional de la República de Costa Rica (7 de abril de 1995). Voto 1887-95. Sentencia: 01887. Expediente: 94-000293-0007-CO. Fecha: 07/04/1995. Hora: 09:15:00 a.m. Emitido por: Sala Constitucional.

Sala Constitucional de la República de Costa Rica (13 de marzo de 1998). Voto 1998-01822. Sentencia: 01822. Expediente: 95-003050-0007CO. Fecha: 13/03/1998. Hora: 10:15:00 a.m. Emitido por Sala Constitucional.

Sistema Nacional de Áreas de Conservación (SINAC). Gobierno de Costa Rica. (2016). Infor- 
me anual estadísticas SEMEC 2016. SINAC en números. $103 \mathrm{p}$.

Silva, J.; Bacao, F. \& Caetano, M. (2017). Specific Land Cover Class Mapping by Semi-Supervised Weighted Support Vector Machines. Rev. Remote Sens. 9:181.

Vargas-Ulate, G. (2009). Turismo y espacios naturales protegidos en Costa Rica: Enfrentamiento o concertación. Rev. Ciencias Sociales 123-124: 49-78 (I-II).

Villegas, J. C. (2012). Informe de levantamiento del Patrimonio Natural del Estado ubicado en Zona Marítimo-Terrestre en área de influencia del $\mathrm{Hu}$ medal Nacional Térraba-Sierpe. Mimeografo. MINAE-ACOSA. Golfito, Costa Rica.
Villegas, J. C. (2013). Informe de levantamiento del Patrimonio Natural del Estado ubicado en la Franja Fronteriza sur de Costa Rica, Área de Conservación Osa. Mimeografo. MINAE-ACOSA. Golfito, Costa Rica.

Yáñez-Arancibia; A. ,Day, J. W.; Twilley, R. R. y Day, R. H. (2014). Manglares: ecosistema centinela frente al cambio climático, Golfo de México. Madera y Bosques 20: 39-75.

Zamora, P. y Cortés, J. (setiembre de 2009). Los manglares de Costa Rica: el Pacífico norte. Rev. Biol. Trop. (Int. J. Trop. Biol. ISSN-00347744)., 57(3): 473-488.

Recibido: : 25 de agosto de 2017 Aceptado: 19 de octubre de 2017 
